\title{
Temporary On-Skin Passive UHF RFID Transfer Tag
}

Mohamed A. Ziai, and John C. Batchelor, Senior Member, IEEE

This is an accepted pre-published version of this paper.

(C) 2011 IEEE. Personal use of this material is permitted. Permission from IEEE must be obtained for all other uses, in any current or future media, including reprinting/republishing this material for advertising or promotional purposes, creating new collective works, for resale or redistribution to servers or lists, or reuse of any copyrighted component of this work in other works.

The link to this paper on IEEE Xplore ${ }^{\circledR}$ is

http://ieeexplore.ieee.org/xpl/articleDetails.jsp?tp=\&arnumber=5978203\&queryText\%3DTemporary+Onskin+Passive+UHF+RFID+Transfer+Tag+Design

The DOI is: $10.1109 /$ TAP.2011.2163789 


\title{
Temporary On-Skin Passive UHF RFID Transfer Tag
}

\author{
Mohamed A. Ziai, and John C. Batchelor, Senior Member, IEEE
}

\begin{abstract}
A passive UHF RFID tag design is presented in the form of a transfer patch similar to a temporary tattoo that is mountable directly onto the skin surface. The transfer tag is suitable for monitoring of people over time in mission critical and secure environments. The antenna reactance is first calculated to conjugate match the measured RFID chip reactance and then full wave simulation is used to design the tag with good performance on a human flesh model. Finally the tag read range is measured on different parts of a volunteer's body and compared to simulated read range values for the entire RFID bands.
\end{abstract}

Index Terms-RFID, Conducting ink, Body Centric Communications

\section{INTRODUCTION}

$\mathrm{W}$ ITH the emergence of distributed and wireless sensor technologies readable tags will be able to collect a vast sea of data that can be processed to provide new information. Such information could be extremely important in mission critical environments such as power plants, airports, military bases and depots, refineries, oil rigs, and access restricted areas to provide the highest quality of security to record trends and take immediate required actions. In these environments as well as health care, monitoring and identifying people is vital to interface different services to create a more resilient system.

Passive RFID is emerging as particularly useful in monitoring, identifying and tracking people in work environments [1-3]. For example, employees or visitors could be located and their action monitored in defined environments over a moderate distance without requiring a deliberate read action from the tagged person [4]. This would allow convenient fast access to restricted areas and a safer working environment for the employee, while benefiting the employing organizations and general public by enhancing security and reliability in the workplace.

More established RFID systems such as LF and HF RFID can be used for the monitoring of people but the person to be identified must come very close to, or even touch, the reader. This is not particularly attractive for many applications where fast access or continuous monitoring is required. UHF RFID systems on the other hand can use relatively high gain standard matched antennas and electromagnetic wave propagation as a coupling mechanism and provide an identification distance (read range) of up to $10 \mathrm{~m}$. The long interrogation range, along with low cost tags and relatively high data rate as shown in Table I make UHF RFID systems suitable for human monitoring, $[5,6]$.

TABLE I

LF, HF AND UHF RFID SYSTEM CHARACTERISTICS [5, 6]

\begin{tabular}{lccc}
\hline \hline & LF & HF & UHF \\
\hline $\begin{array}{l}\text { Typical RFID } \\
\text { Frequency }\end{array}$ & $125-134 \mathrm{kHz}$ & $13.56 \mathrm{MHz}$ & $865-956 \mathrm{MHz}$ \\
$\begin{array}{l}\text { Approximate read } \\
\text { distance }\end{array}$ & $>0.5 \mathrm{~m}$ & $>0.7 \mathrm{~m}$ & $0.5-10 \mathrm{~m}$ \\
$\begin{array}{l}\text { Typical data- } \\
\text { transfer rate }\end{array}$ & $1 \mathrm{kbit} / \mathrm{s}$ & $25 \mathrm{kbit} / \mathrm{s}$ & $30 \mathrm{kbit} / \mathrm{s}$
\end{tabular}

\section{RFID TAGS FOR HUMAN MONITORING}

Human tagging, external to the body, is usually based on wrist bands or ID badges which could be removed and given to other people. There is little work reported on skin mounted RFID tags that cannot be removed without destroying the tag, and [7-11] describe work which requires a substantial gap of several millimeters between the tag and the skin to provide an acceptable read range. These designs are also complicated by multiple layers either with or without cross layer connections, and therefore they are not suitable for ultra low profile tags which can be directly mounted on skin.

Being a passive system, the power collected by the tag antenna is used to activate the tag IC. The transmitted read power available to the tag antenna is constrained by electromagnetic compatibility regulations and thus it is essential that the maximum collected power is transferred to the IC as shown by the Friis equation [12]:

$$
R \leq \frac{\lambda}{4 \pi} \sqrt{\frac{E I R P_{\text {reader }} \times G_{\text {tag }} \times \eta \times \tau \times \rho}{P_{\text {th }}}}
$$

where, $E I R P_{\text {reader }}$ is the effective isotropic radiated power of the reader; $G_{t a g}$ is the tag antenna gain; $P_{t h}$ is the ASIC circuit activation threshold power; $\eta$ is the efficiency of the rectifier; $\tau$ is impedance matching coefficient between the antenna and the ASIC; and $\rho$ is the polarization coefficient between the reader and tag antenna.

The polarization loss factor is given as [13]:

$$
\rho=\left|\hat{\rho}_{w} \cdot \hat{\rho}_{a}\right|^{2}
$$

where, $\hat{\rho}_{w}$ is the incoming wave polarisation orientation and $\hat{\rho}_{a}$ is the polarisation of the receiving antenna. 
Even though most RFID reader antennas are circularly polarized, the orientation plane of the receiving antenna or tag on the body is unknown (as it varies with body position), and therefore polarization loss could potentially be high causing a reduction in read range.

Also coupling to the human body adversely affects the tag antenna gain, and consequently reduces the power available to the chip.

Since in designing a tag for human identification, the tag designer has no control over tag IC rectifier efficiency and has little control over tag polarization loss, therefore maximizing the power transmission coefficient $\tau$ to the chip is very important. The power transmission coefficient is given as [14]:

$$
\tau=1-|\Gamma|^{2}=\frac{4 R_{a} R_{I C}}{\left(R_{a}+R_{I C}\right)^{2}+\left(X_{a}+X_{I C}\right)^{2}},
$$

where: $0 \leq \tau \leq 1$ and $\Gamma=\frac{z_{I C}-Z_{a}^{*}}{z_{I C}+z_{a}}, 0<|\Gamma|<1$

where $\Gamma$ is the reflection coefficient between the antenna and the ASIC input impedances; $R_{a}$ and $R_{I C}$ represent the antenna and the chip input resistances; and $X_{a}$ and $X_{I C}$ are the antenna and the chip input reactance, respectively. Therefore, a conjugate match $\left(Z_{I C}=Z_{a}^{*}\right)$ is required for maximum power transfer from the tag antenna to the IC which is significantly capacitive.

Being in close proximity to body tissues which have significant conductivity and permittivity the tag is capacitively loaded and the antenna radiation resistance is reduced. This presents a challenge to the designer to achieve high tag input inductance with a small antenna which is suitable for use on a human body. Currently reported designs have a thickness of about $0.015 \lambda(1.8-4 \mathrm{~mm})$ to achieve an acceptable efficiency $[7,10,15]$.

This paper describes a substrate-less nested slotline antenna with design guidelines suitable for use directly on the skin as a transfer tattoo, i.e. no dielectric substrate or air space is required between the tag metallization and the surface of the skin. The tag is formed from a single conducting layer and therefore requires no via connections.

\section{TRANSFER RFID TAG DESIGN}

Fig.1. shows a nested slotline antenna [15] that produces a surface current on a rectangular conductive patch. The antenna is designed to radiate at the RFID UHF band. The slot is formed mid-length along the patch and a small distance $t$ from the upper edge. Two short coplanar lines separated by gap $G$ extend from the slot centre point out of the edge of the patch and the RFID ASIC is connected across the two lines as shown in Fig.1. An electric field is induced in the slot which causes an electric current loop to flow around it. This thin loop provides an inductance to cancel the negative reactance of the capacitive ASIC. Since the slot width is very narrow compared to wavelength, and the current is not confined to the edge of the slot, it spreads out over the conductive patch and the antenna effective aperture size is large enough to offer an improved radiation efficiency, [16]. The tag acts as an electric dipole, linearly polarized along the $x$-axis.

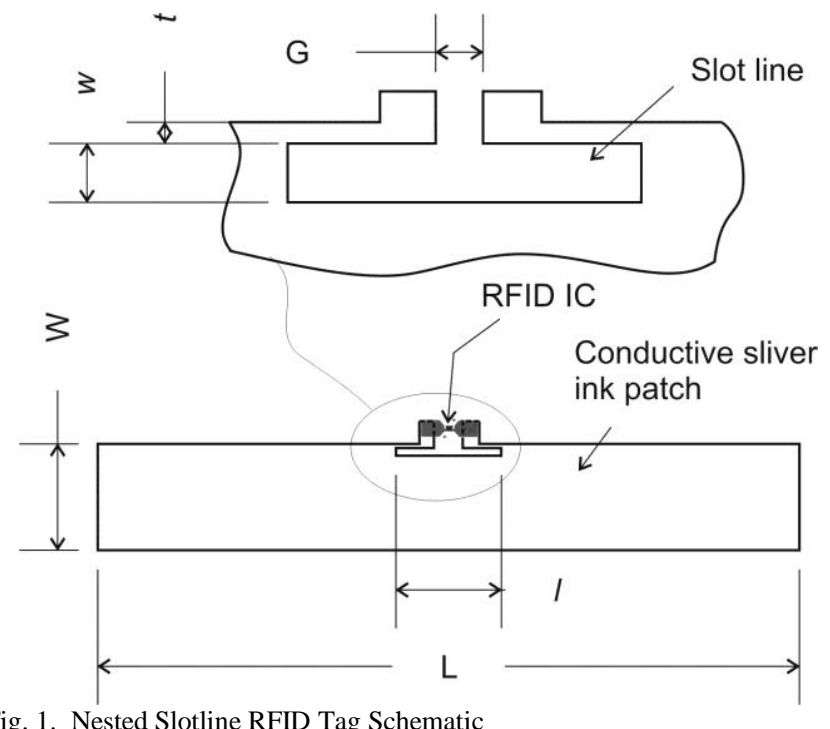

Fig. 1. Nested Slotline RFID Tag Schematic

The nested slotline antenna resonant frequency and port reactance is controlled by the electrical length of the slot line, where the slotline resonant length and reactance can be approximated using Cohn's closed form expressions [17] as shown below.

The equivalent circuit of the packaged IC connected to the antenna is shown in Fig.2, where the IC is represented as the voltage source " $V_{g}$ " and capacitive impedance " $Z_{g}$ ".

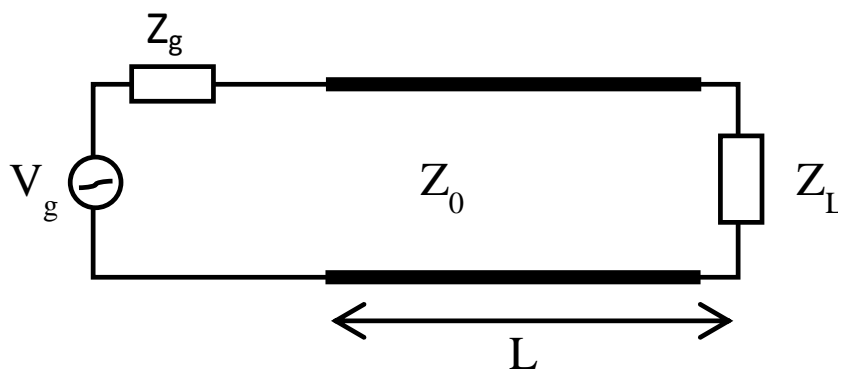

Fig. 2. Slotline equivalent circuit.

The conjugate matched impedance between the antenna and the RFID chip is represented by:

$Z_{I C}=Z_{I N}^{*}$

where $Z_{I N}$ is the input impedance for a transmission line of length $l$, and characteristic impedance of $Z_{0}$, terminated on load $Z_{L}$, given by [18]:

$$
Z_{I N}=Z_{0} \frac{\left[Z_{L} \cos (\beta l)+j Z_{0} \sin (\beta l)\right]}{\left[Z_{0} \cos (\beta l)+j Z_{L} \sin (\beta l)\right]}
$$

where $Z_{0}$ can be calculated for slotline of width $w$ on a substrate of height $h$. If the ratio of $0.2 \leq w / h \leq 1$ [18]:

$Z_{0}=113.19-53.55 \log \varepsilon_{\mathrm{r}}+1.25 w / h\left(114.59-51.88 \log \varepsilon_{\mathrm{r}}\right)+$ $20(w / h-0.2)(1-w / h)-\left[0.15+0.23 \log \varepsilon_{\mathrm{r}}+w / h(-0.79+\right.$ $\left.\left.2.07 \log \varepsilon_{\mathrm{r}}\right)\right] \cdot\left[10.25-5 \log \varepsilon_{\mathrm{r}}+w / h\left(2.1-1.42 \log \varepsilon_{\mathrm{r}}\right)-h / \lambda_{0} \times\right.$ $100]^{2}$ 
The effective dielectric constant of the slotline $\varepsilon_{r}$ is less than the substrate permittivity $\varepsilon$, and can be calculated using the following equation:

$$
\varepsilon_{r}=\frac{\varepsilon+1}{2}+1
$$

The propagation constant $\beta$ for an ideal system without any losses is given by [17]:

$$
\beta=\left(2 \pi / \lambda_{0}\right) \sqrt{\left(1 / \lambda_{s} / \lambda_{0}\right)^{2}-1}
$$

where $\lambda_{0}$ and $\lambda_{s}$ denote the free space and slotline wavelength and the ratio $\lambda_{0} / \lambda_{s}$ is given by [17][1]:

$$
\begin{aligned}
\lambda_{s} / \lambda_{0}= & 0.987-0.485 \log \varepsilon_{r}+w / h\left(0.111-0.002 \varepsilon_{r}\right)- \\
& \left(0.121+0.094 w / h-0.0032 \varepsilon_{r}\right) \log \left(h / \lambda_{0} \times 100\right)
\end{aligned}
$$

To ascertain appropriate values for $\varepsilon$ in (7) and $h$ in (6) and (9) a simulated study using CST Microwave Studio ${ }^{\mathrm{TM}}$ was carried out using the Transient Solver Finite Difference Time Domain (FDTD) method. Simulation of a slotline on a dielectric block at $915 \mathrm{MHz}$ indicated that a very simple homogenous flesh model with $\varepsilon=15$ and conductivity $\sigma=0.255 \mathrm{~s} / \mathrm{m}$ was sufficient to represent the human surface which is in agreement with the results of [7]. Simulated fields were observed to penetrate no deeper than $10 \mathrm{~mm}$, so this value was used for $h$ in the closed form equations. The above expressions are then used to calculate the slotline dimensions that provide a conjugate match to the RFID IC reactance. As the tag is mounted directly onto skin with no intervening dielectric sheet, the electrical parameters of flesh are required for the substrate calculation. Here we design the tag for the NXP G2XL flip chip strap package with quoted typical port impedance 14.8$\mathrm{j} 125 \Omega$.

TABLE II

Nested SlotLine Dimensions on Homogenous Human Tissue Block

$\begin{array}{cccc}\text { Parameters } & w(\mathrm{~mm}) & l(\mathrm{~mm}) & h(\mathrm{~mm}) \\ 3 & 14.5 & 10\end{array}$

Therefore, assuming no losses, a slotline antenna on a $10 \mathrm{~mm}$ homogenous substrate with parameters given in Table II gives an input impedance of $Z_{I N}=j 125 \Omega$ at $915 \mathrm{MHz}$ which is appropriate to cancel the manufacturer's quoted input reactance. Having calculated the approximate value of the slotline antenna, the patch size can also be approximated on an homogenous substrate. As the conductive patch length affects the current distribution it should be long enough to avoid disturbing the high density of the currents around the slotline. It also affects the antenna gain, and higher gains are achieved for resonant patch size. Therefore the conductive patch resonant length at $915 \mathrm{MHz}$ was calculated using a first order approximation for a half wave patch length given by [19]:

$$
L=\frac{c}{2 f_{r} \sqrt{\varepsilon_{r}}}
$$

where $c$ is the speed of light in vacuum and $f_{r}$ is the resonant frequency of the antenna. The patch width is chosen to be wide enough not to curtail the surface currents and is at least 3 times the slot width.

The slotline parameters obtained in Table I and the calculated patch size are then modeled in CST using the transient FDTD solver to guide the design of a matched and tuned tag directly onto skin with no intervening substrate. For improved accuracy, rather than use the homogenous model which gave the initial slotline and patch length, a 4 layer model of human tissue is created in CST using the electrical data given in Table III, [20].

TABLE III

LAYERED HuMAN MOdEL ELECTRICAL PARAMETERS AT 900MHz, [20]

\begin{tabular}{lccc}
\hline \hline Layer & $\varepsilon_{r}$ & $\sigma(\mathrm{S} / \mathrm{m})$ & $\begin{array}{c}\text { Layer thickness } \\
(\mathrm{mm})\end{array}$ \\
\hline Skin and fat & 14.5 & 0.25 & 5 \\
Muscle & 55 & 0.94 & 10 \\
Bone & 12.6 & 3.85 & 5 \\
Internal Organs & 52 & 0.91 & 20
\end{tabular}

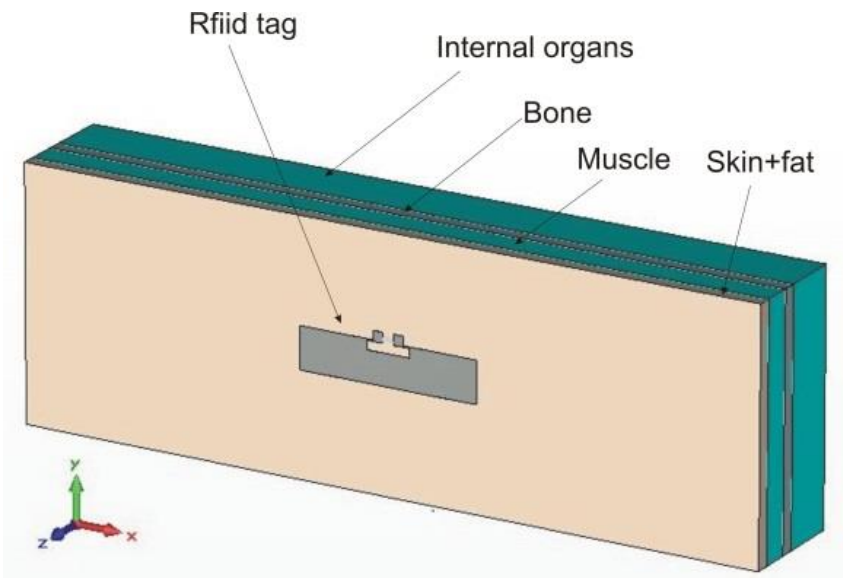

Fig. 3. RFID transfer tag mounted on multilayer human model.

Table IV lists the simulated values of the antenna parameters optimized for power transfer, gain, and bandwidth on the human torso model.

TABLE IV

SimUlated SLOTLINE DimENSIONS OBTAINED ON LAYERED PHANTOM

\begin{tabular}{cccccc}
\hline \hline Parameters (mm) & $L$ & $W$ & $l$ & $\mathrm{w}$ & $\mathrm{t}$ \\
\hline & 65 & 20 & 14.5 & 3 & 0.5
\end{tabular}

Parameters are defined in Fig.1.

\section{Transfer Tag Design Procedure}

The fields in the slot dominate the reactive impedance presented to the chip. Therefore the input impedance is mostly influenced by the slot length $l$ and width $w$. The resistive part of the tag impedance is significantly affected by the slot size, and the thickness of the feed lines $t$ which significantly alters the current flow into the chip. The half wave dipole patch currents are not strong compared to the current intensity around the ASIC feed point and slot transformer and therefore, providing the patch remains relatively wide, the patch size does not strongly affect the tag performance other than to have a secondary influence on resonant frequency. The gain of the tag is determined by the patch size. 
To inform the general design process, Figs.4-6 show parameter curves obtained from simulation. For a given chip input match and desired frequency, Fig.4 allows a suitable slot length $l$ to be obtained and combined with a related slot width $w$.

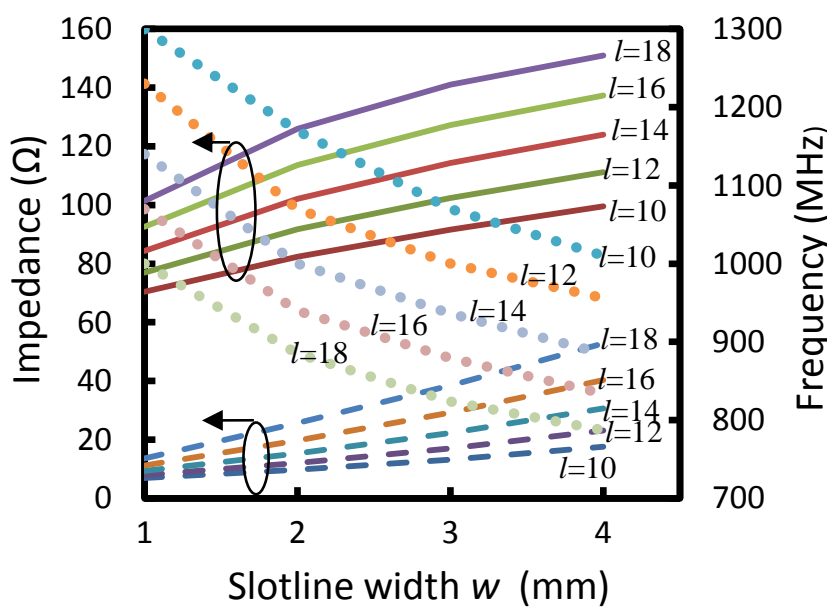

Fig. 4. Effect of slot length $l$ and width $w$ on antenna port impedance and resonant frequency. Broken lines $=$ resistance, Solid lines $=$ reactance dotted lines $=$ resonant frequency.

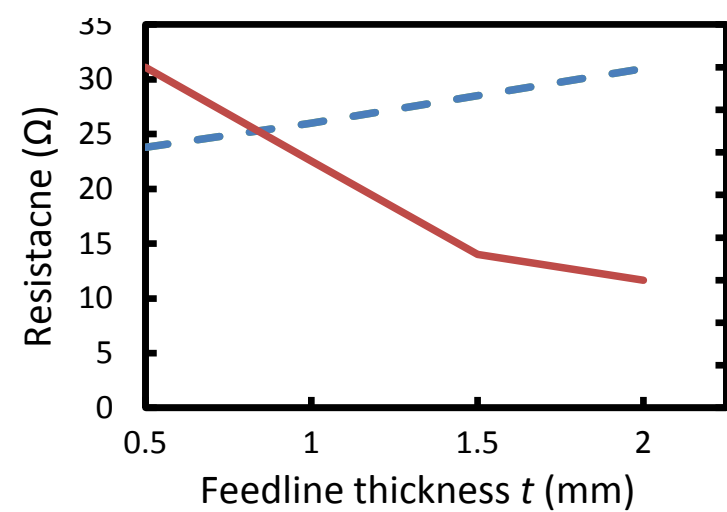

118 118 117 117 孚 116 올 116 115 Ч 115 \& 114 114

Fig. 5. Effect of feedline thickness $t$ on antenna port impedance and resonant frequency. Broken line $=$ resistance, Solid line $=$ reactance.

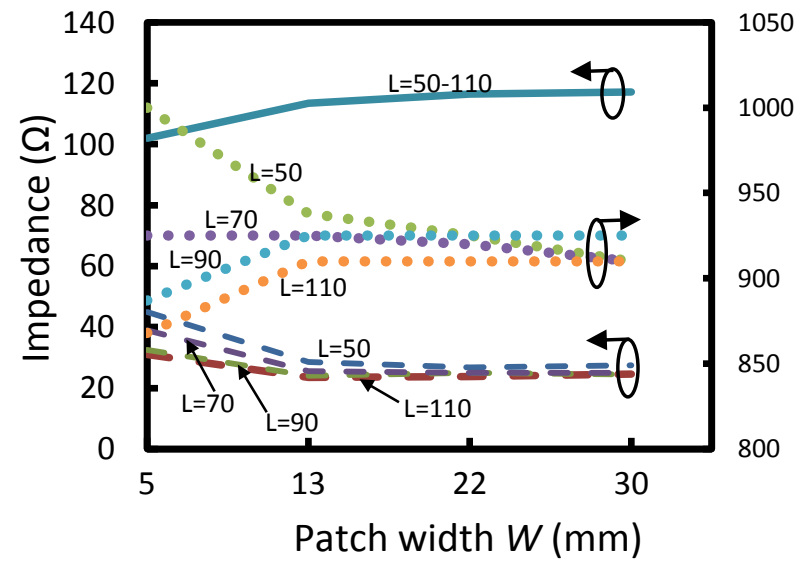

Fig. 6. Effect of patch width $W$ and length $L(\mathrm{~mm})$ on antenna port impedance and resonant frequency. Broken lines $=$ resistance, Solid lines $=$ reactance, dotted lines $=$ resonant frequency.

Fig.5 relates the line thickness $t$ to input impedance, while Fig.6 relates patch dimensions $L$ and $W$ to input impedance and operating frequency. The antenna reactance is primarily determined by the slotline length $l$ meaning the following steps can be taken to design the antenna:

1- Based on the quoted or measured capacitance of the ASIC, the length of the slot line is calculated using equations 5-9 to generate the required inductance to cancel the capacitive reactance of the ASIC and any parasitic effects of the chip fixture.

2- Using equation 10, patch length $L$ is calculated for resonance at $915 \mathrm{MHz}$. The patch width is selected to be equal to the slot length $l$ to reduce disruption to the fields around the slotline.

3- For a given slot length $l$, the slot line width $w$ is then selected from Fig.4 to resonate at close to the center resonant frequency $(915 \mathrm{MHz})$. The slot acts as an input transformer and strongly affects the tag terminal impedance and as a consequence, the resonant frequency.

4- The Feed line width $t$ is selected to tune the real part of the antenna impedance (Fig.5). The antenna resonant frequency is not sensitive to this parameter and the effect on tag reactance is only secondary.

5- Further tuning can be performed by adjusting the patch size to tune the antenna impedance (Fig.6). The patch is essentially a wide half wave dipole with distributed current surface currents. As shown in Fig.6, these low density currents on the patch do not greatly affect the resonant frequency providing the patch is not too narrow, in which case it becomes a thin dipole with an input impedance more strongly affected by its length. The antenna gain variation with patch dimensions is shown in Fig.7. Higher gains are obtained for those lengths where half wave modes are supported and provided the patch is not too narrow, the gain is not strongly influenced by patch width (gain variations of $1 \mathrm{~dB}$ or less are simulated for a $25 \mathrm{~mm}$ change in width).

Using the design curves of Figs.4-7, a value of the port impedance $(14+j 114 \Omega)$ can be achieved by selecting the parameters listed in Table 2. This provides a reasonable match to the quoted NXP G2XL impedance of $14.8-\mathrm{j} 125 \Omega$.

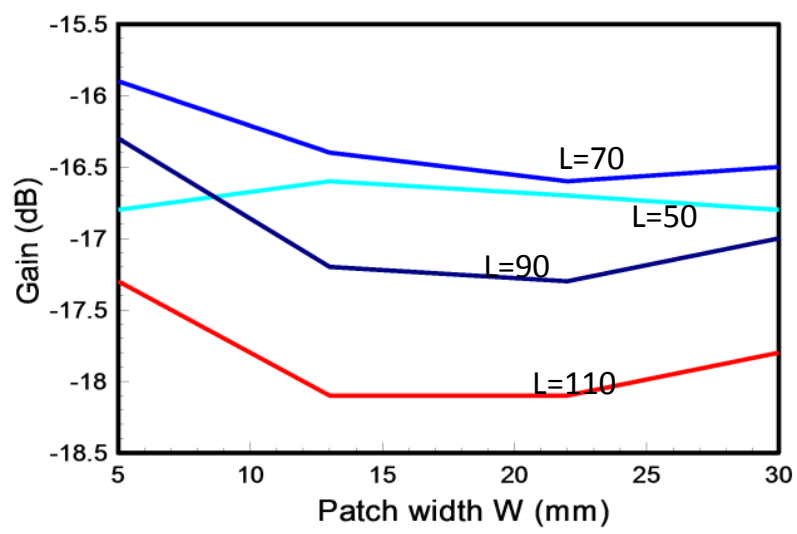

Fig. 7. Effect of patch width $\mathrm{W}$ and length $\mathrm{L}(\mathrm{mm})$ on the antenna gain.

\section{RADIATION PATTERN AND SURFACE CURRENT DISTRIBUTION}

A simulated polar plot of the slotline antenna directivity on the layered human model is shown in Fig.8 where the maximum directivity is squinted around $40^{\circ}$ from bore-site. This squint 
arises because the feed is not located on the center line of the finite sized human tissue block. The squint should not be evident when the tag is mounted on a continuous skin surface. The maximum directivity of the tag on the human model block is $4 \mathrm{dBi}$ but radiation efficiency is very low on the human phantom $(-20.7 \mathrm{~dB})$ which as expected reduces the gain and consequently tag read range according to the Friis free space equation (1).

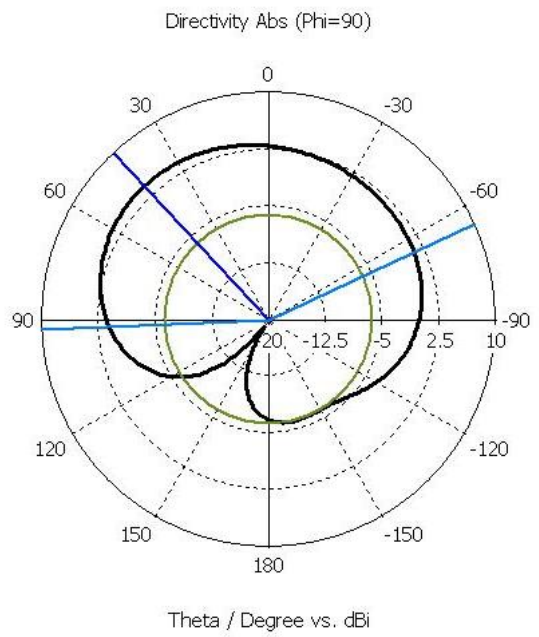

Fig. 8. Polar plot of simulated antenna directivity in $y-z$ plane when mounted on human model.

Fig.9 shows the surface current distribution of the antenna on the human model. Strong surface currents are located close to the edge of the slotline and they spread out on the patch surface creating a half wave resonance along the patch.

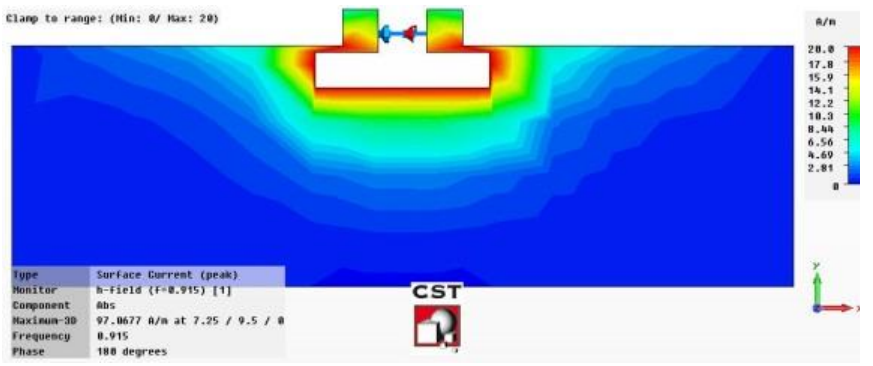

Fig. 9. Simulated surface current distribution of tag on human model

Fig.10 illustrates the simulated slotline antenna resonant frequency and bandwidth on the human model. The slot line antenna has an input return loss of $20 \mathrm{~dB}$ at the center resonance frequency $(915 \mathrm{MHz})$, with enough bandwidth (119MHz from $864-983 \mathrm{MHz}$ ) to cover the entire FCC RFID frequency bands at an input return loss of $10 \mathrm{~dB}$.

\section{FABRICATION AND IMPEDANCE MEASUREMENT}

A prototype tag was fabricated using the parameters given in Table 2. The antenna structure pattern was first etched as a negative in a thin metal stencil. The metalized layer was deposited on temporary transfer tattoo inkjet paper [27] using electrically conductive silver paint [28] which was profiled using the stencil. The paper covering layer of the Temporary Tattoo Transfer material was removed leaving the electrically

conductive silver paint antenna on a thin layer $(\sim 10 \mu \mathrm{m})$ of plastic.

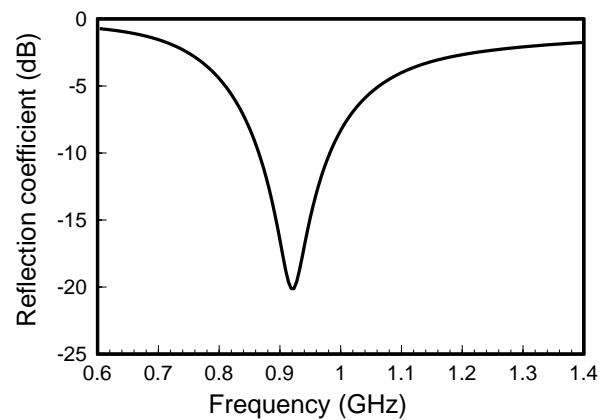

Fig. 10 Simulated reflection coefficient in $\mathrm{dB}$ against frequency in $\mathrm{GHz}$ for tag on human model.

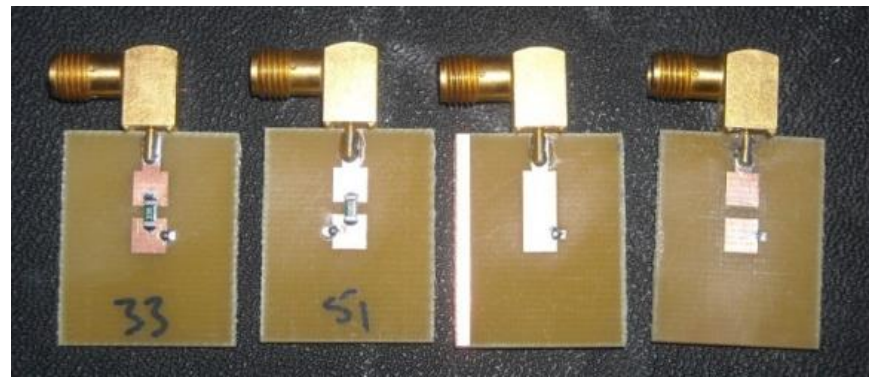

Fig. 11. RFID chip impedance measurement kit.

The transfer tattoo tag was then placed on a volunteer's forearm to measure the impedance at the port contacts. The port impedance was measured using a Bazooka balun [21] tuned at $910 \mathrm{MHz}$ to transform the unbalanced vector network analyzer (VNA) coaxial cable to the balanced input required at the antenna port. In this measurement the VNA [22] was calibrated to the end of the coaxial cable using a standard calibration kit, and then the calibrated reference plane was offset to the tip of the probe. The measured tag port impedance was $Z_{a}=27+j 97$.

To maximize the power transfer from the antenna to the chip, the input impedance of the chip with its fixing straps was also measured. This was achieved using five bespoke test calibration boards, [23], as shown in Fig.11. Three of the boards were designed to calibrate the VNA using the shortopen-load technique. The load board contained a $50 \Omega$ resistor. The fourth test board was used to measure the resistivity of a known resistor to evaluate the calibration (in this case $30 \Omega$ ), while the fifth test board was used to measure the RFID chip impedance. The VNA was calibrated using the calibration kit and verified using the $30 \Omega$ load with $\pm 1 \%$. The RFID chip with contact strap impedance was fixed to the test board using electrically conductive adhesive transfer tape [24] and the measured chip impedance is compared to the quoted typical manufacture's value in Table $\mathrm{V}$.

TABLE $\mathrm{V}$

MEASURED AND QUOTED NXP G2XL ASIC PORT IMPEDANCE AT 915MHz

Quoted Manufacturer typical impedance

Measured port impedance

The RFID IC was then attached to the transfer tattoo tag ports using electrically conductive adhesive transfer tape and the $14.8-\mathrm{j} 125 \Omega$

$16-\mathrm{j} 119 \Omega$ 
prototype tag was transferred directly to a volunteer's skin as shown in Fig. 12.

\section{READ RANGE MEASUREMENTS}

The tattoo transfer tag read range was measured on the volunteers forearm, stomach and chest, using a monostatic reader with $27.5 \mathrm{dBm}$ ERP using an AS3990 reader chipset, [25] connected to a $6.5 \mathrm{dBic}$ circularly polarized patch antenna via a short coax cable. All tag read ranges were measured in a lab environment and are compared to simulated read ranges assuming tag and the reader antennas have matching polarization

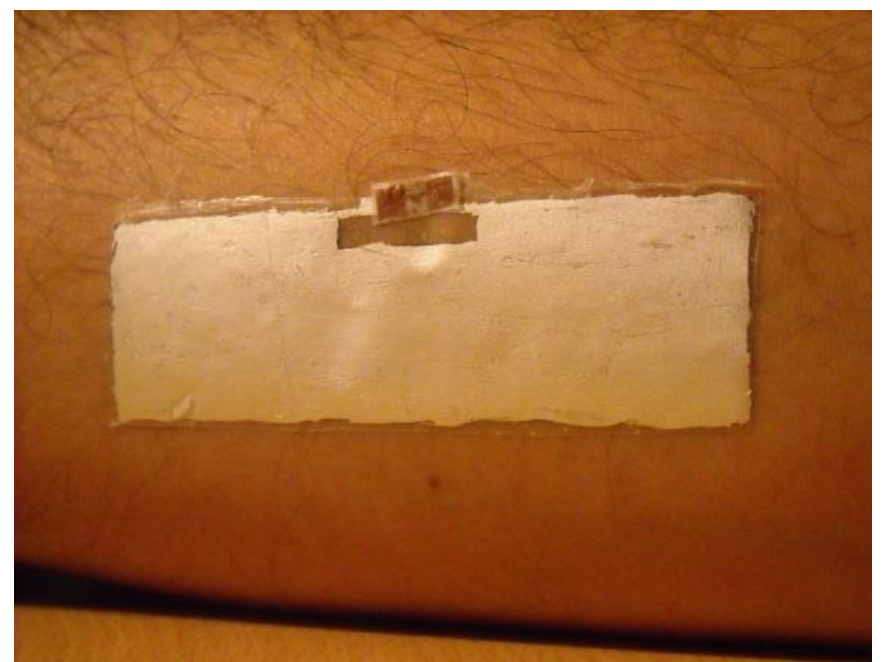

Fig. 12. Tattoo transfer tag mounted on volunteer arm. Conducting paint separated from skin by a 10 micrometer layer.

The prototype tattoo tags were placed on the forearm, stomach and chest of a volunteer and the maximum ranges at which tags read in the bands for different countries were measured on many occasions and the average distances calculated to mitigate against multipath effects. As illustrated in Fig.13 the measured prototype tag read range on the forearm was $80 \mathrm{~cm}$, lower than simulated (about $100 \mathrm{~cm}$ ) while the measured read range on the chest $(110 \mathrm{~cm})$ and stomach $(120 \mathrm{~cm})$ was higher than simulation indicated. This is because the forearm has a thinner layer of skin and fat than assumed in simulation. The tag antenna was initially tuned on a model representing the thorax with thicker skin and fat layers so the prototype tag was less efficient on the forearm than simulation predicted. Higher losses due to higher conductivity and permittivity of the dense muscles and bone in the forearm could also reduce the antenna radiation efficiency compared to mounting sites on the thorax. The prototype tag durability was tested by wearing the prototype tattoo transfer tag on the volunteer's forearm throughout a working day and regularly testing its functionality by measuring read range for the duration of 5 hours. The read range measured was found to be consistent during the experiment period with the volunteer engaged in normal office activities.

\section{CONCLUSIONS AND FURTHER DEVELOPMENTS}

This paper has introduced a new concept of placing ultra thin RFID tags directly onto the skin in the form of a transfer tattoo. The tag is a novel topology of a slotted patch which requires no artificial substrate layer between the metallization and the human skin surface. Results indicated that useful read ranges of $80 \mathrm{~cm}$ are possible when mounted on a forearm and increasing to $120 \mathrm{~cm}$ when mounted on the thorax. These read ranges are impressive considering the tag is mounted directly on the skin with only a $10 \mu \mathrm{m}$ thick adhesive layer. A design procedure for the tag is provided and with refinement the tag can be optimized to give higher read ranges specific parts of the human body. Applications are proposed for temporary security clearance, ticketing and military use or where it might not be desirable to insert sub-skin RFID capsules. Although RFID has been used to illustrate the concept, it is envisaged that skin transfer antennas could also be designed with the terminals connected by surgical plaster. The technology could also be applicable for medical sensing and as a pick up for implanted devices. Inkjet conducting ink processes could make this a cheap and widespread technology, [26].

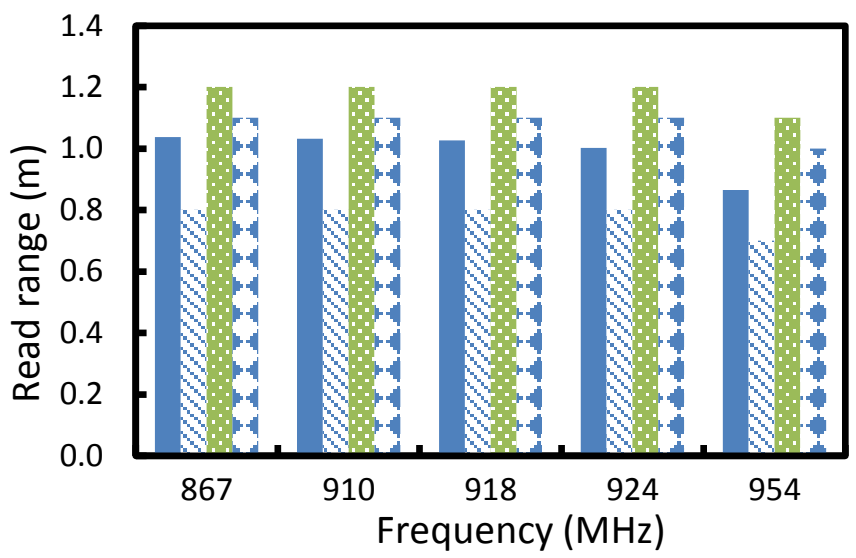

Fig. 13. Simulated and measured read range for tattoo tag mounted on human. Solid blue bar $=$ simulated on human model, crossed blue bar $=$ measured on volunteer forearm, dotted green line $=$ measured on the volunteer stomach and blue dimond bar $=$ measured on volunteer chest. $867 \mathrm{MHz}, 910 \mathrm{MHz}$, $918 \mathrm{MHz}, 924 \mathrm{MHz}, 954 \mathrm{MHz}$ represent center FCC RFID frequency bands for Europe, USA, Korea, China and Japan respectively.

\section{ACKNOWLEDGMENT}

We thank Austriamicrosystems for providing the RFID reader development kit.

\section{REFERENCES}

[1] Intelleflex_RFID. Personnel Monitoring. Available: http://www.intelleflex.com/Solutions.PM.asp

[2] Motorola RFID. Motorola's healthcare mobility solutions, "Improving patient safety at the point of care in theatre and in hospitals". Available: http://www.motorola.com/web/Business/Solutions/Federal\%20Governm ent/_Documents/Static\%20files/Fed_Healthcare_AB_FINAL.pdf?locale $\mathrm{Id}=33$

[3] H. Lehpamer, "RFID design principles," Artech House Microwave Library, p. 293, 2007. ISBN-13: 978-1596931947

[4] C. Chao, et al., "Improving patient safety with RFID and mobile technology," International Journal of Electronic Healthcare, vol. 3, pp. 175-192, 2007.

[5] Z. Chen, Antennas for portable devices: John Wiley \& Sons, Inc. New York, NY, USA, 2007. ISBN-13: 978-0470030738

[6] Atmel. Considerations for RFID selection. Available: http://www.atmel.com/dyn/resources/Prod_documents/secrerf_3_04.pdf

[7] G. Marrocco, "Body-matched RFID antennas for wireless biometry," 2006, pp. 1-5. Antennas and Propagation. EuCAP 2006. First European Conference on. ISBN: 92-9092-937-5 
[8] C. Occhiuzzi, et al., "Body-matched Slot Antennas for RadioFrequency Identification." XXIX URSI General Assembly. 7-16 August, 2008. Chicago, USA

[9] G. Marrocco, "RFID antennas for the UHF remote monitoring of human subjects," Antennas and Propagation, IEEE Transactions on, vol. 55, pp. 1862-1870, 2007.

[10] H. Rajagopalan, Y. Rahmat-Samii, "Conformal RFID Antenna Design Suitable for. Human Monitoring and Metallic Platforms," Antennas and Propagation (EuCAP), 2010 Proceedings of the Fourth European Conference on . 12-16 April 2010, page(s): 1 - 5, ISBN:978-1-42446431-9.

[11] M. Ziai and J. Batchelor, "Thin ultra high-frequency platform insensitive radio frequency identification tags," Microwaves, Antennas \& Propagation, IET, vol. 4, pp. 390-398, 2010.

[12] K. Finkenzeller, RFID Handbook: Fundamentals and Applications in Contactless Smart Cards and Identification: Wiley, 2003. ISBN-13: 9780471988519

[13] C. Balanis, Antenna theory: Wiley New York, 1997. ISBN-13: 9780471592686.

[14] Rao, K.V.S.; Nikitin, P.V.; Lam, S.F., "Impedance matching concepts in RFID transponder design,", Automatic Identification Advanced Technologies. Fourth IEEE Workshop on, pp. 39-42. Issue Date: 17-18 Oct. 2005, ISBN: 0-7695-2475-3

[15] G. Marrocco, "RFID Antennas for the UHF Remote Monitoring of Human Subjects," Antennas and Propagation, IEEE Transactions on, vol. 55, pp. 1862-1870, 2007.

[16] J. Kraus and R. Marhefka, Antennas: McGraw-Hill New York, 1988. ISBN: 0070354227

[17] K. C. Gupta, Microstrip Lines and Slotlines 2nd Ed: Artech House Publishers; 2 edition, 1996. ISBN-13: 978-0890067666

[18] B. Wadell, Transmission line design handbook: Artech House Boston (Ma), 1991. ISBN-13: 978-0890064368

[19] A. Ittipiboon, R. Garg, Inder Bahl, P. Bhartia, "Microstrip Antenna Design Handbook, Artech House," Inc. 2001. ISBN-13: 9780890065136

[20] C Gabriel, S Gabriel and E Corthout, "The dielectric properties of biological tissues: I. Literature survey," Physics in Medicine and Biology, vol. 41, p. 2231, 1996.

[21] K. Fujimoto, Mobile antenna systems handbook: Artech House Publishers, 2008. ISBN-13: 978-1596931268

[22] Anritsu. Anritsu ME7808 broadband Vector Network Analyzer. Available: http://www.anritsu.co.uk/

[23] L. Mayer and A. Scholtz, "Sensitivity and impedance measurements on UHF RFID transponder chips," The Second International EURASIP Workshop on RFID Technology, 7-8 July, 2008, Budapest, Hungary

[24] M. E. C. T. 9703. 3M $\mathrm{M}^{\mathrm{TM}}$ Electrically Conductive Tape 9703. Available: http://solutions.3m.com/wps/portal/3M/en_WW/Graphics/Scotchprint/Pr od-

Info/Catalog/?PC_7_RJH9U5230GE3E02LECIE20S7M3_nid=23L97PJ H4CbeGX9B4321ZWgl

[25] Austriamicrosystems. UHF RFID and HF RFID Reader IC manufacturer. Available: http://www.austriamicrosystems.com/

[26] J. Batchelor, et al., "Inkjet printing of frequency selective surfaces," Electronics Letters, vol. 45, pp. 7-8, 2008.

[27] TheMagicTouch. "Temporary Tattoo Transfer Paper". Available: http://www.themagictouch.co.uk/transfer/tattoo.htm

[28] Electrolube. "Silver Conductive Paint". Available: http://www.electrolube.com/docs/catalog.pdf

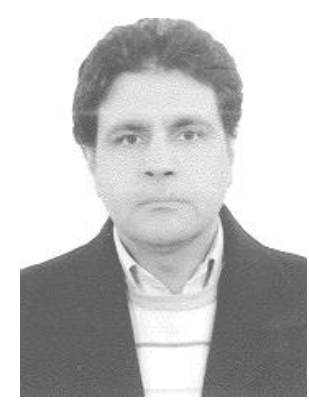

M. Ali Ziai received the MSc in Broad band and Wireless Communication Engineering from the University of Kent, Canterbury, U.K., and is currently working toward the Ph.D. degree in electromagnetic fileds at the Kent University.

His main research activities are aimed at the Electrically Thin and Platform Insensitive Ultra High Frequency Radio Frequency Identification Tags. His other research interests are linked to the field of antennas and UHF RFID.
John C. Batchelor (S'93-M'95-SM'07) received the B.Sc. and Ph.D. degrees from the University of Kent, Canterbury, U.K., in 1991 and 1995, respectively.

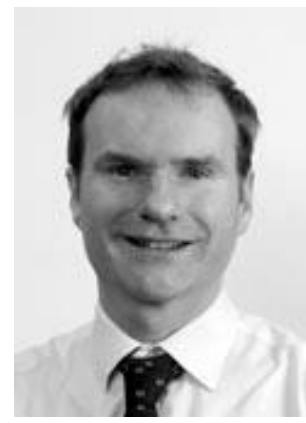

From 1994 to 1996 , he was a Research Assistant with the Electronics Department, University of Kent, and in 1997, became a Lecturer of electronic engineering. He now leads the Antennas Group at Kent and is a Reader in Antenna Technology. His current research interests include UHF RFID tag design, body-centric antennas, printed antennas, compact multiband antennas, electromagnetic bandgap structures, and long-wavelength FSS (frequency-selective surfaces). 
AP1012-1375.R1 